\title{
High frequency of voriconazole-related phototoxicity in cystic fibrosis patients
}

To the Editors:

Aspergillus fumigatus infection is common in cystic fibrosis (CF) patients and estimates of the rate of allergic bronchopulmonary aspergillosis range from $1-15 \%$. Voriconazole, a new triazole antifungal, has been recognised as efficient and safe in the treatment of invasive aspergillosis [1-2]. Since it is available orally, voriconazole became the first alternative to itraconazole. We began giving voriconazole to CF patients in 2003, and we encountered an unexpectedly high frequency of moderate-tosevere skin reactions, which limited the administration of the drug.

We therefore reviewed all the medical files of children (aged $<18$ yrs; $n=72)$ and adults $(n=63)$ followed up in our tertiary $\mathrm{CF}$ reference centre between April 2003 and December 2008, in order to estimate the frequency of voriconazole-induced photosensitivity, as previously defined [3]. All the patients who were given voraconazole were selected. Demographic data, cystic fibrosis transmembrane conductance regulator (CFTR) mutations, clinical data, treatment, duration and voriconazole dosage, and related adverse effects (AEs) were collected. Qualitative and quantitative comparisons were analysed using the Chi-squared test and nonparametric Mann-Whitney test, respectively. Multivariate analyses were performed by using stepwise logistic regression.
A p-value $<0.05$ was considered statistically significant. Statistical analyses were performed using Statistica 8 (StatSoft, MaisonsAlfort, France).

$31(21.4 \%)$ out of the $135 \mathrm{CF}$ patients were given voriconazole. Their characteristics are described in table 1 . When genotypes were categorised into CFTR mutation classes, 36.5\% and 58\%, respectively, were heterozygous and homozygous for class II mutations, which alter the maturation of CFTR protein. Voriconazole-related AEs were reported in 19 (61\%) patients. One patient suffered from a persistent dazzle. All others presented with photosensitivity reactions such as facial rash, moderate-to-severe sunburn or fine desquamation of exposed skin. Eight $(44.5 \%)$ received voriconazole during the winter, and all were recommended to apply skin protection at the time of prescription. The onset time of photosensitivity was available in children and ranged from 22-349 days (median 146 days). 11 $(61 \%)$ patients required interruption of treatment because of these reactions.

The frequency of AEs was not significantly different in children $(63 \%)$ versus adults $(50 \%)$ or in males $(66 \%)$ versus females $(50 \%)$. As expected, the daily dose $\left(\mathrm{mg} \cdot \mathrm{kg}^{-1}\right)$ given was higher in the paediatric population than in the adult one: median 9 (range 5.0 24.2) $\mathrm{mg} \cdot \mathrm{kg}^{-1}$ versus 8 (5.2-9.3) $\mathrm{mg} \cdot \mathrm{kg}^{-1}(\mathrm{p}=0.034)$.

\section{TABLE 1 Description of the whole population exposed to voriconazole and comparison regarding photosensitivity groups}

\begin{tabular}{|c|c|c|c|c|}
\hline & Total population & Photosensitivity & No photosensitivity & p-value \\
\hline Subjects n & 31 & 18 & 13 & \\
\hline Age yrs & $15.8(4-35)$ & $13.5(4-26.1)$ & $16.8(6.75-35)$ & 0.08 \\
\hline Age $<18$ yrs & 19 (61.3) & $12(66.7)$ & $7(53.8)$ & 0.28 \\
\hline Sex male/female $n$ & $15 / 16$ & $8 / 10$ & $8 / 5$ & 0.60 \\
\hline$\Delta \mathrm{F} 508 / \Delta \mathrm{F} 508$ & $13(41.9)$ & $12(66.7)$ & $1(7.7)$ & \\
\hline$\Delta \mathrm{F} 508 /$ other & $16(51.6)$ & $5(27.8)$ & $11(84.6)$ & \\
\hline Other/other & $2(6.5)$ & $1(5.6)$ & $1(7.7)$ & \\
\hline CFTR mutation (class II) & & & & 0.024 \\
\hline Class II/class II & $18(58.1)$ & $14(77.8)$ & $4(30.8)$ & \\
\hline $\mathrm{mg}$ & $400(120-400)$ & $400(120-400)$ & $400(200-400)$ & \\
\hline $\mathrm{mg} \cdot \mathrm{kg}^{-1}$ & $8(5.0-24.2)$ & $9(1.3-28)$ & $8(2.5-64)$ & 0.10 \\
\hline Time of drug exposure months & $6.5(1.3-64)$ & $6.5(1.3-28)$ & $15(2.5-64)$ & 0.44 \\
\hline $\begin{array}{l}\text { Treatment interruption in relation with skin } \\
\text { reactions }\end{array}$ & $11(35.5)$ & $11(61.1)$ & 0 & \\
\hline
\end{tabular}


Photosensitivity was strongly associated with $\Delta \mathrm{F} 508 / \Delta \mathrm{F} 508$ genotype (OR 24, 95\% CI 2.5-230.7; $\mathrm{p}=0.004$ ) but was not with pancreatic insufficiency. The association remained when the analysis was extended to class II genotype (OR 6.3, 95\% CI 1.6$52.6 ; \mathrm{p}=0.01)$. The group with photosensitivity tended to be younger, and further analysis showed that seven out of the 12 concerned children were aged less than 9 yrs. Multivariate analysis showed that photosensitivity was only independently associated with $\Delta \mathrm{F} 508 / \Delta \mathrm{F} 508$ genotype (OR 24.5, 95\% CI $2.2-$ $272.9 ; \mathrm{p}=0.009)$. Seven patients were also given ciprofloxacin, and only one of them belonged to the photosensitivity group.

This survey observed a high frequency (58\%) of photosensitivity among $31 \mathrm{CF}$ patients treated with voriconazole. Voriconazole has been considered as a well-tolerated drug in large cohorts of patients with various immunity statuses. Skin eruptions [1] have been described in 0.5 to $5.3 \%$ of cases in various phase III trials [4], and recently up to $8.3 \%$ in an open study on chronic aspergillosis treatment [5]. On the other hand, voriconazole-related photosensitivity has rarely been reported in CF children or adult patients [2,6-7]. This high rate of photosensitivity was not in line with the previous reported ratio of $14 \%$ in CF children [2], but closer to $40 \%$ in lung-transplanted CF adults [6]. A recent Canadian case report raised the question of voriconazole safety as five out of the six CF children treated with voriconazole presented photosensitivity. By comparison, WALSH et al. [8] reported $16 \%$ of cutaneous AEs in 69 non-CF children, and only three $(4.3 \%)$ were identified as photosensitive. The patients from our CF centre were given prolonged voriconazole treatment, which might influence the $\mathrm{AE}$ rate. The mean duration of treatment was close to 1 year in our patients, which was comparable to that reported by BERGE et al. [6] (9.6-22 months) and CHENG et al. [7] (6-22 months), but longer than that reported by HiLLIARD et al. [2] ( $<29$ weeks). We nevertheless noticed that photosensitivity could occur at any time during the treatment; i.e. from a few weeks to $1 \mathrm{yr}$ in our survey. The occurrence of voriconazole-related photosensitivity was related neither to the length of the treatment nor to the administered dose. Although not assessed in this survey, a relationship with high serum levels appeared unlikely as previously shown [6-8]. Lastly, ciprofloxacin, well known to induce photosensitivity, was excluded as an interfering risk factor.

The mechanism of voriconazole-related photosensitivity remains unknown. Voriconazole is metabolised by the human cytochrome P-450 and its isoenzymes, mainly CYP2C19 and to a lesser extent CYP2C9 and CYP3A4, but no relationship has yet been demonstrated between this pathway and photoreactions [1]. Thus, BERGE et al. [6] have recently proven that CYP2C19 genotypes influence voriconazole exposure without any impact on the occurrence of AEs [6]. Voriconazole-related skin reactions have been assimilated to retinoid-like sensitivity [3], as retinoid acid and metabolites induced photosensitivity and high serum levels were measured in three out of the five reported adults [5]. DENNING and GRIFFITHS [9] hypothesised that voriconazole might inhibit a step in the metabolic breakdown of all-trans retinol (vitamin A) through the cytochrome P-450 pathway that they shared. This led to the establishment of a possible link with the common vitamin A supplementation required in pancreatic insufficiency CF patients [7]. Although all the patients were indeed vitamin A supplemented, pancreatic insufficiency was not identified in our study as an associated factor for photosensitivity, contrary to the strong relationship established with the $\Delta \mathrm{F} 508 / \Delta \mathrm{F} 508$ genotype. This association has never been underlined, and it might be of interest that the genotype of the three patients with photosensitivity reported by HILLIARD et al. [2] was also $\Delta \mathrm{F} 508 / \Delta \mathrm{F} 508$. Otherwise, ciprofloxacin-related photosensitivity has been stated as uncommonly appearing, with a high frequency in CF [10]. This suggests that CF disease itself might be a risk factor to facilitate the expression of drug-related photosensitivity, and would agree with a possible ability of voriconazole or one of its metabolites to elicit a phototoxic reaction, which is a common mechanism. $\Delta \mathrm{F} 508 / \Delta \mathrm{F} 508$ mutation alters the maturation of the CFTR protein, which is an ATPbinding cassette transporter (ABC, subfamily $\mathrm{C}$ ) and a $\mathrm{CAMP}$ regulated $\mathrm{Cl}^{-}$channel that mediates transepithelial $\mathrm{Cl}^{-}$transport in various tissues. CFTR has been shown to limit reactive oxygen species (ROS) production. Interestingly, both ABC transporters [11] and ROS [12] have been implicated in phototoxicity mechanisms. This pathway could however not sustain the whole mechanism of voriconazole-induced photosensitivity that we observed. The genotype analysis of the patients with no skin reaction showed that all but two of them nevertheless carried two mutations deeply altering the CFTR protein either through its maturation or its production.

In conclusion, CF patients appeared highly photosensitive to voriconazole, independently of seasonality and despite the recommended skin protection that we provided. This should be taken into account by $\mathrm{CF}$ practitioners, as several case reports have warned on the association of prolonged voriconazole therapy with discoid lupus erythematosus-like lesions or secondary squamous cell carcinoma $[8,13]$. Otherwise, the involvement of the $\Delta$ F508 CFTR mutation might be helpful for further research in the understanding of these common adverse drug effects.

\section{S. Rondeau*, L. Couderc*, S. Dominique ${ }^{\#}$, S. Pramil ${ }^{\#}$,} C. Leguillon*,\#, B. Masseline ${ }^{*, \#}$, L. Favennec ${ }^{*}$ and C. Marguet* ${ }^{*} \mathrm{CF}$ Reference Centre (CRCM), Dept of Paediatrics, ${ }^{\#} \mathrm{CF}$ Reference Centre (CRCM), Dept of Pulmonology, Charles Nicolle University Hospital, and "Microbiology Dept, Mycology Unit, Charles Nicolle University Hospital, EA3830 Rouen University, Rouen, France.

Correspondence: C. Marguet, CF Reference Centre, Pulmonology and Allergy, Dept of Paediatrics, Charles Nicolle University Hospital, Rouen University, 1 rue de Germont, 76031 Rouen, France. E-mail: christophe.marguet@chu-rouen.fr

Statement of Interest: None declared.

\section{REFERENCES}

1 Johnson LB, Kauffman CA. Voriconazole: a new triazole antifungal agent. Clin Infect Dis 2003; 36: 630-637.

2 Hilliard T, Edwards S, Buchdahl R, et al. Voriconazole therapy in children with cystic fibrosis. J Cyst Fibros 2005; 4: 215-220.

3 Rubenstein M, Levy ML, Metry D. Voriconazole-induced retinoidlike photosensitivity in children. Pediatr Dermatol 2004; 21: 675-678.

4 Pfizer Canada. Voriconazole Product Monograph. www.pfizer.ca/ en/our_products/products/monograph/140 Date last updated: August, 4: 2010. 
5 Denning DW, Ribaud P, Milpied N, et al. Efficacy and safety of voriconazole in the treatment of acute invasive aspergillosis. Clin Infect Dis 2002; 34: 563-571.

6 Berge M, Guillemain R, Tregouet DA, et al. Effect of cytochrome P450 2C19 genotype on voriconazole exposure in cystic fibrosis lung transplant patients. Eur J Clin Pharmacol 2011; 67: 253-260.

7 Cheng MP, Paquette K, Lands LC, et al. Voriconazole inhibition of vitamin A metabolism: are adverse events increased in cystic fibrosis patients? Pediatr Pulmonol 2010; 45: 661-666.

8 Walsh TJ, Karlsson MO, Driscoll T, et al. Pharmacokinetics and safety of intravenous voriconazole in children after single- or multiple-dose administration. Antimicrob Agents Chemother 2004; 48: 2166-2172.

9 Denning DW, Griffiths CE. Muco-cutaneous retinoid-effects and facial erythema related to the novel triazole antifungal agent voriconazole. Clin Exp Dermatol 2001; 26: 648-653.
10 Burdge DR, Nakielna EM, Rabin HR. Photosensitivity associated with ciprofloxacin use in adult patients with cystic fibrosis. Antimicrob Agents Chemother 1995; 39: 793.

11 Tamura A, An R, Hagiya Y, et al. Drug-induced phototoxicity evoked by inhibition of human ABC transporter ABCG2: development of in vitro high-speed screening systems. Expert Opin Drug Metab Toxicol 2008; 4: 255-272.

12 Agrawal N, Ray RS, Farooq M, et al. Photosensitizing potential of ciprofloxacin at ambient level of UV radiation. Photochem Photobiol 2007; 83: 1226-1236.

13 McCarthy KL, Playford EG, Looke DF, et al. Severe photosensitivity causing multifocal squamous cell carcinomas secondary to prolonged voriconazole therapy. Clin Infect Dis 2007; 44: e55-e56.

DOI: 10.1183/09031936.00097611 